\title{
A Super-Integrable Two-Dimensional Non-Linear Oscillator with an Exactly Solvable Quantum Analog
}

\author{
José F. CARIÑNEA ${ }^{\dagger}$, Manuel F. RAÑADA ${ }^{\dagger}$ and Mariano SANTANDER ${ }^{\ddagger}$ \\ † Departamento de Física Teórica, Facultad de Ciencias \\ Universidad de Zaragoza, 50009 Zaragoza, Spain \\ E-mail:jfc@unizar.es,mfran@unizar.es \\ ¥Departamento de Fúsica Teórica, Facultad de Ciencias \\ Universidad de Valladolid, 47011 Valladolid, Spain \\ E-mail:msn@fta.uva.es
}

Received October 31, 2006, in final form January 24, 2007; Published online February 24, 2007

Original article is available at http://www.emis.de/journals/SIGMA/2007/030/

\begin{abstract}
Two super-integrable and super-separable classical systems which can be considered as deformations of the harmonic oscillator and the Smorodinsky-Winternitz in two dimensions are studied and identified with motions in spaces of constant curvature, the deformation parameter being related with the curvature. In this sense these systems are to be considered as a harmonic oscillator and a Smorodinsky-Winternitz system in such bi-dimensional spaces of constant curvature. The quantization of the first system will be carried out and it is shown that it is super-solvable in the sense that the Schrödinger equation reduces, in three different coordinate systems, to two separate equations involving only one degree of freedom.
\end{abstract}

Key words: deformed oscillator; integrability, super-integrability; Hamilton-Jacobi separability; Hamilton-Jacobi super-separability; quantum solvable systems

2000 Mathematics Subject Classification: 37J35; 34A34; 34C15; 70H06

\section{Super-integrable systems}

There are few integrable systems in the Arnold-Liouville sense: Hamiltonian systems in a $2 n$ dimensional symplectic manifold for which there exist $n$ functionally independent constants of motion $f_{i}$ in involution (including the Hamiltonian $H$ itself), i.e.

$$
\left\{f_{i}, f_{j}\right\}=0, \quad \forall i, j=1, \ldots, n, \quad d f_{1} \wedge \cdots \wedge d f_{n} \neq 0
$$

The system is said to be super-integrable when it is integrable and there exists a set of $m>n$ functionally independent constants of motion, i.e. it possesses more independent first integrals than degrees of freedom. The existence of these additional first integrals gives rise to a higher degree of regularity in the phase space (e.g. there exist periodic orbits) since the trajectories are restricted to submanifolds of dimension lower than $n$. In particular, a system with $n$ degrees of freedom possessing $2 n-1$ independent first integrals is said to be maximally super-integrable.

It is also well-known [1] that the only central potentials in which all bounded orbits are closed (periodic) are given by $V=(1 / 2) \omega_{0}^{2} r^{2}$ and $V=-k / r$. From a modern point of view the existence of closed trajectories is considered as a consequence of the existence of the maximal number of functionally independent integrals of motion; thus, the result obtained by Bertrand

${ }^{\star}$ This paper is a contribution to the Proceedings of the Workshop on Geometric Aspects of Integrable Systems (July 17-19, 2006, University of Coimbra, Portugal). The full collection is available at http://www.emis.de/journals/SIGMA/Coimbra2006.html 
is a proof of the super-integrability of these two systems. In fact, the harmonic oscillator shares with the Kepler problem a very distinguished property not only in Classical but also in Quantum Mechanics, where super-integrability leads to energy levels depending on a single quantum number.

Fris et al. studied in 1965 the Euclidean $n=2$ systems which admit separability in two different coordinate systems [2], and obtained four families $V_{r}, r=a, b, c, d$, of super-integrable potentials with constants of motion linear or quadratic in the velocities (momenta). The two first families

$$
V_{a}=\frac{1}{2} \omega_{0}^{2}\left(x^{2}+y^{2}\right)+\frac{k_{2}}{x^{2}}+\frac{k_{3}}{y^{2}}, \quad V_{b}=\frac{1}{2} \omega_{0}^{2}\left(4 x^{2}+y^{2}\right)+k_{2} x+\frac{k_{3}}{y^{2}},
$$

can be considered as the more general Euclidean deformations (with strengths $k_{2}, k_{3}$ ) of the $1: 1$ and $2: 1$ harmonic oscillators preserving quadratic super-integrability (the other two families, $V_{c}$ and $V_{d}$, were related with the Kepler problem). The super-integrability of $V_{a}$, which is known as the 'Smorodinsky-Winternitz' (S-W) potential, has been studied by Evans [3, 4] for the general case of $n$ degrees of freedom.

A large number of papers have been published on super-integrability in these last years, most of them related with quadratic superintegrabilty (see [5] for the proceedings of a workshop on super-integrability and Refs. [6, 7, 8, 9] for some very recent studies on super-integrable systems with integrals quadratic in momenta). The idea is that if we call super-separable a system that admits Hamilton-Jacobi separation of variables (Schrödinger in the quantum case) in more than one coordinate system, then quadratic super-integrability (i.e., super-integrability with linear or quadratic constants of motion) can be considered as a property arising from super-separability. We note that these studies also include non-Euclidean Hamiltonian systems $[10,11,12,13,14,15,16,17,18,19,20,21,22]$ and that in both cases, Euclidean and nonEuclidean, many of these systems are closely related with the harmonic oscillator.

The rareness of integrable systems leads to the following question. Is it possible to deform a super-integrable system but preserve super-integrability of the system? We report here some results of previous works where such question is affirmatively answered. We shall show that this is possible for two important examples: the isotropic bi-dimensional harmonic oscillator and the Smorodinsky-Winternitz system.

A very interesting example of a one-dimensional nonlinear oscillator depending of a parameter $\lambda$ was studied in 1974 by Mathews and Lakshmanan [23, 24] and it has recently been proved [25] that this particular nonlinear system can be generalized to the two-dimensional case, and even to the $n$-dimensional case: these higher dimensional systems are super-integrable deformations admitting $2 n-1$ quadratic constants of motion. It is also proved that there is a related $\lambda$-dependent two-dimensional oscillator isotonic oscillator that is a super-integrable deformation, i.e. a $\lambda$-dependent version of the Smorodinski-Winternitz system [26, 27]. Actually, the deformation introduced by the parameter $\lambda$ modifies the Hamilton-Jacobi equation but preserves the existence of a multiple separability. Moreover, we point out that a geometric interpretation of the higher-dimensional systems was proposed in relation with the dynamics on spaces of constant curvature.

This paper must be considered as a survey summarizing recent works by the authors on properties related with the integrability and super-integrability of certain two-dimensional $\lambda$-dependent systems related with the harmonic oscillator and it is neither a review of the whole field nor a comparison with other approaches of different authors. It is mainly focused on the study of deformations of super-integrable systems that do not alter the super-integrability structure. It starts with a brief review of the basic properties of the above mentioned isotonic oscillator, $\mathrm{S}-\mathrm{W}$ system and Mathews-Lakshmanan oscillator and some two-dimensional $\lambda$-dependent oscillatorlike systems, first in in the classical approach and afterwards in their quantum counterparts. 
Roughly speaking this survey is divided into three main parts: first, the analysis of the classical $\lambda$-dependent oscillator including the existence of Hamilton-Jacobi multiple separability (existence of alternative coordinate systems in which the corresponding $\lambda$-dependent HamiltonJacobi equation separates). Second, the relation of this $\lambda$-dependent nonlinear model with the harmonic oscillator on the three spaces of constant curvature $\left(S^{2}, \mathbb{E}^{2}, H^{2}\right)$. The final part is devoted to the analysis of these systems from the quantum viewpoint.

The second part presents a geometrical approach and proves that these systems can be considered in two different ways: either as a nonlinear deformation of a linear system, or simply as a model of the oscillator on spaces of constant curvature. In the first case the parameter $\lambda$ represents the strength of the deformation and in the second one the curvature of the space. Finally, in the third part, devoted to the quantum version of this $\lambda$-dependent oscillator, it is proved that the system is exactly solvable: the Hamiltonian can be factorized and the wave functions and energies can be explicitly obtained. It includes some points such as: (i) Analysis of the transition from the classical $\lambda$-dependent system to the quantum one, (ii) Exact resolution of the $\lambda$-dependent Schrödinger equation, factorization method and existence of operators $A$ and $A^{+}$, and shape-invariance property, (iii) Schrödinger multiple separability and quantum super-integrability.

In more detail the structure of the paper is as follows: In next section we describe shortly two one-dimensional classical systems which can be considered as generalizations of the harmonic oscillator and the isotonic oscillator: the $\lambda$-deformed nonlinear oscillator and the deformed isotonic oscillator. Section 3 deals with two-dimensional generalizations of these classical systems and their deformations and Section 4 studies the separability properties of their Hamilton Jacobi equations. A geometric interpretation is given in Section 5 and a quantization of the nonlinear oscillator, is carried out in Section 6, and the quantum spectrum s computed by using the traditional power series expansion. The system is shown in Section 7 to admit a shape invariant factorization which allows us to explicitly compute the spectrum in an algebraic alternative way. Finally, in Section 8 we sketch the method to be used for the corresponding two-dimensional system and prove that he Hamiltonian can be written as a sum of three different terms such that each one commutes with the sum of the other two, what provides us alternative complete sets of compatible observables.

\section{Some one-dimensional classical systems}

We start this section by reviewing the basic properties of two simple classical systems.

\subsection{The harmonic oscillator}

The dynamics of the classical harmonic oscillator in one dimension is given by

$$
\frac{d x}{d t}=v, \quad \frac{d v}{d t}=-\omega^{2} x
$$

and is described by a Lagrangian $L=(1 / 2)\left(v^{2}-\omega^{2} x^{2}\right)$. A complex variable $z=\omega x+i v$ may be introduced and the equations of the motion become

$$
\frac{d z}{d t}=-i \omega z
$$

whose general solution is $z=z_{0} e^{-i \omega t}$, i.e.

$$
x=x_{0} \cos \omega t-\frac{v_{0}}{\omega} \sin \omega t=A \cos (\omega t+\varphi),
$$

and therefore the solutions are periodic with angular frequency $\omega$, while $A$ and $\varphi$ are arbitrary. 


\subsection{The isotonic harmonic oscillator}

The isotonic oscillator is described by the Lagrangian [28, 29]

$$
L=\frac{1}{2} v_{x}^{2}-\frac{1}{2} \alpha^{2} x^{2}-\frac{k}{x^{2}}, \quad k>0,
$$

(like a harmonic oscillator plus a centripetal barrier). This is an important example of an isochronous system and actually they are the only two rational potentials giving rise to isochronous systems [30, 31].

The Euler-Lagrange equation

$$
\ddot{x}+\alpha^{2} x+\frac{c}{x^{3}}=0, \quad c=-2 k,
$$

is a particular case of the so-called Pinney-Ermakov equation [32, 33] whose general solution can be written as $x=\left(1 /(\alpha A) \sqrt{\left(\alpha^{2} A^{4}+c\right) \sin ^{2}(\alpha t+\phi)-c}\right.$.

The corresponding quantum system admits a shape-invariant factorization [34] and therefore is solvable by means of algebraic methods.

\subsection{A 1-dimensional nonlinear oscillator}

In 1974 Mathews and Lakshmanan [23] studied the equation of motion

$$
\left(1+\lambda x^{2}\right) \ddot{x}-\lambda x \dot{x}^{2}+\alpha^{2} x=0, \quad \lambda>0 .
$$

The general solution takes the form $x=A \sin (\omega t+\phi)$, with the following additional restriction linking angular frequency $\omega$ and amplitude $A$ :

$$
\omega^{2}=\frac{\alpha^{2}}{1+\lambda A^{2}}
$$

The equation (1) is the Euler-Lagrange equation for the Lagrangian:

$$
L_{\lambda}(x, \dot{x})=\frac{1}{2} \frac{1}{1+\lambda x^{2}}\left(\dot{x}^{2}-\alpha^{2} x^{2}\right) .
$$

It describes a system with nonlinear oscillations with an amplitude dependent frequency (or period). We can also allow negative values for $\lambda$ [25], but when $\lambda<0$ the values of $x$ are limited by the condition $|x|<1 / \sqrt{|\lambda|}$. In the limit $\lambda \rightarrow 0$ we recover the equation of motion and both the Lagrangian of the harmonic oscillator and the frequency become independent of the amplitude. Therefore, the system can be seen as a deformation of the harmonic oscillator.

It can also be seen as an oscillator with a position-dependent effective mass which depends on $\lambda$ :

$$
m_{\lambda}=\frac{1}{1+\lambda x^{2}}
$$

The Hamiltonian for such a system is:

$$
H_{\lambda}(x, p)=\frac{1}{2}\left(1+\lambda x^{2}\right) p^{2}+\frac{1}{2} \frac{\alpha^{2} x^{2}}{1+\lambda x^{2}} .
$$

The important fact is that there is an interesting generalization to $n=2$ or even arbitrary $n$ [25].

Note that the Lagrangian for the one-dimensional free-particle (i.e. for $\alpha=0$ )

$$
L_{\lambda}\left(x, v_{x}\right)=T_{1}(\lambda)=\frac{1}{2} \frac{v_{x}^{2}}{1+\lambda x^{2}}
$$


is invariant under the vector field

$$
X_{x}^{t}(\lambda)=\sqrt{1+\lambda x^{2}} \frac{\partial}{\partial x}+\frac{\lambda x v_{x}}{\sqrt{1+\lambda x^{2}}} \frac{\partial}{\partial v_{x}} .
$$

which is the natural lift to the phase space $\mathbb{R} \times \mathbb{R}$ of the vector field in $\mathbb{R}$

$$
X_{x}(\lambda)=\sqrt{1+\lambda x^{2}} \frac{\partial}{\partial x}
$$

i.e. $X_{x}^{t}(\lambda)\left(T_{1}(\lambda)\right)=0$.

\subsection{A deformed isotonic oscillator}

We study next a deformed isotonic oscillator in one dimension described by the Lagrangian [26]:

$$
L_{\lambda}\left(x, v_{x}, k\right)=\frac{1}{2} \frac{v_{x}^{2}}{1+\lambda x^{2}}-\frac{1}{2} \frac{\alpha^{2} x^{2}}{1+\lambda x^{2}}-\frac{k}{x^{2}} .
$$

Here $\lambda$ can be any real number, but when $\lambda<0$ the possible values of $x$ are such that $|x|<$ $1 / \sqrt{|\lambda|}$.

The Euler-Lagrange equation is

$$
\frac{d^{2} x}{d t^{2}}-\frac{\lambda x}{1+\lambda x^{2}}\left(\frac{d x}{d t}\right)^{2}+\frac{\alpha^{2} x}{1+\lambda x^{2}}-2 k \frac{1+\lambda x^{2}}{x^{3}}=0
$$

and one can see that the general solution for bounded motions is [26]:

$$
x=\frac{1}{\omega A} \sqrt{\left(\omega^{2} A^{4}-2 k\right) \sin ^{2}(\omega t+\phi)+2 k},
$$

where

$$
\omega R_{1}\left[2 k+\left(\omega^{2} A^{4}-2 k\right) \sin ^{2}(\omega t+\phi)\right]^{2}=0
$$

with

$$
R_{1}=\lambda \omega^{2} A^{4}-\left(\alpha^{2}-\omega^{2}-2 k \lambda^{2}\right) A^{2}+2 k \lambda .
$$

It can be done similarly for unbounded motions, the functions $\sin (\omega t+\phi)$ being then replaced by functions $\sinh (\Omega t+\phi)$. There also exist limit unbounded motions of the form

$$
x=\sqrt{(A t+B)^{2}+C} .
$$

It will be shown that as in the harmonic oscillator case, there is an interesting generalization to the $n=2$ case.

\section{Some two-dimensional classical systems}

\subsection{The harmonic oscillator}

The Hamiltonian of a two-dimensional classical harmonic oscillator is (for simplicity $m=1$ ):

$$
H\left(x, y, p_{x}, p_{y}\right)=\frac{1}{2}\left(p_{x}^{2}+p_{y}^{2}\right)+\frac{1}{2}\left(\omega_{1}^{2} x^{2}+\omega_{2}^{2} y^{2}\right),
$$


and we can easily check that the energy functions for each degree of freedom are constants of motion:

$$
I_{1}=E_{x}=\frac{1}{2}\left(p_{x}^{2}+\omega_{1}^{2} x^{2}\right), \quad I_{2}=E_{y}=\frac{1}{2}\left(p_{v}^{2}+\omega_{2}^{2} y^{2}\right) .
$$

The rational case, for which $\omega_{1}=n_{1} \omega_{0}, \omega_{2}=n_{2} \omega_{0}$, with $n_{1}, n_{2} \in \mathbb{N}$, is super-integrable [35]. In fact, let $K_{x}$ and $K_{y}$ be defined by $K_{x}=p_{x}+i n_{1} \omega_{0} x, K_{y}=p_{y}+i n_{2} \omega_{0} y$. The Hamiltonian $H$ and the canonical symplectic form $\Omega_{0}$ can be expressed in terms of such functions as follows:

$$
H=\frac{1}{2}\left(K_{x} K_{x}^{*}+K_{y} K_{y}^{*}\right), \quad \Omega_{0}=\frac{i}{2 n_{1} \omega_{0}} d K_{x} \wedge d K_{x}^{*}+\frac{i}{2 n_{2} \omega_{0}} d K_{y} \wedge d K_{y}^{*},
$$

and therefore, as the fundamental Poisson brackets are $\left\{K_{x}, K_{x}^{*}\right\}=2 i n_{1} \omega_{0}$ and $\left\{K_{y}, K_{y}^{*}\right\}=$ $2 i n_{2} \omega_{0}$, the evolution equations are

$$
\frac{d}{d t} K_{x}=i n_{1} \omega_{0} K_{x}, \quad \frac{d}{d t} K_{y}^{*}=-i n_{2} \omega_{0} K_{y}^{*} .
$$

Hence, the complex function $J$ defined as $J_{n_{1}, n_{2}}=K_{x}^{n_{2}}\left(K_{y}^{*}\right)^{n_{1}}$ is a (complex) constant of motion which determines two different real first integrals: $I_{3}=\operatorname{Im}\left(J_{n_{1}, n_{2}}\right)$ and $I_{4}=\operatorname{Re}\left(J_{n_{1}, n_{2}}\right)$. They are polynomials in the momenta of degrees $n_{1}+n_{2}-1$ and $n_{1}+n_{2}$, respectively.

Two particularly important examples are the isotropic harmonic oscillator (for $n_{1}=n_{2}=1$ ) and the case $n_{1}=1, n_{2}=2$. In these cases the four constants are not independent but only three of them are independent. For instance, in the isotropic case the constants of motion take the form $I_{3}=p_{x} p_{y}+\omega^{2} x y$ and $I_{4}=x p_{y}-y p_{x}$.

\subsection{The Smorodinsky-Winternitz system}

A 2-dimensional generalization of the isotonic oscillator with rotational symmetry would be

$$
V_{r i}=\frac{1}{2} \omega_{0}^{2}\left(x^{2}+y^{2}\right)+\frac{k_{1}}{x^{2}+y^{2}} .
$$

There is however another super-integrable generalization given by the Smorodinsky-Winternitz potential [2]

$$
V_{S W}=\frac{1}{2} \omega_{0}^{2}\left(x^{2}+y^{2}\right)+\frac{k_{2}}{x^{2}}+\frac{k_{3}}{y^{2}},
$$

which is a set of two non-interacting isotonic systems with the same frequency and in general different constants $k_{2} \neq k_{3}$. Of course, the case $k_{2}=k_{3}=0$ reduces to the usual isotropic harmonic oscillator. The energies of each degree of freedom are constants of the motion and there exists a third constant of motion given by

$$
C=J^{2}+k_{2} \frac{y^{2}}{x^{2}}-k_{3} \frac{x^{2}}{y^{2}}, \quad \text { with } \quad J=x v_{y}-y v_{x} .
$$

\subsection{A nonlinear oscillator in two dimensions}

It has recently been proved [25] that there exists a 1-parameter dependent generalization of the nonlinear oscillator for the 2-dimensional case with the following requirements:

1. The kinetic term $T_{2}(\lambda)$ is a quadratic function of the velocities invariant under rotations.

2. $T_{2}(\lambda)$ is invariant under (the tangent lifts of) the vector fields $X_{1}(\lambda)$ and $X_{2}(\lambda)$ given by

$$
X_{1}(\lambda)=\sqrt{1+\lambda r^{2}} \frac{\partial}{\partial x}, \quad X_{2}(\lambda)=\sqrt{1+\lambda r^{2}} \frac{\partial}{\partial y},
$$

which are extensions to $\mathbb{R}^{2}$ of the vector field $X_{x}(\lambda)$ in the $n=1$ case given by (2). 
These conditions lead to the following form for the kinetic energy:

$$
T_{2}(\lambda)=\frac{1}{2} \frac{1}{1+\lambda r^{2}}\left[v_{x}^{2}+v_{y}^{2}+\lambda\left(x v_{y}-y v_{x}\right)^{2}\right], \quad r^{2}=x^{2}+y^{2} .
$$

The term $\lambda\left(x v_{y}-y v_{x}\right)^{2}$ represents a two-dimensional contribution that cannot appear in the one-dimensional case. When $\lambda<0$ this function will have a singularity at $1-|\lambda| r^{2}=0$ and we should restrict our dynamics to the interior of the circle $x^{2}+y^{2}<1 /|\lambda|$ where $T_{2}(\lambda)$ is positive definite.

The kinetic energy $T_{2}(\lambda)$ is determined by the $\lambda$-dependent metric

$$
d s^{2}(\lambda)=\frac{1}{1+\lambda r^{2}}\left[\left(1+\lambda y^{2}\right) d x^{2}+\left(1+\lambda x^{2}\right) d y^{2}-2 \lambda x y d x d y\right] .
$$

$T_{2}(\lambda)$ remains invariant under the actions of the lifts of the vector fields $X_{1}(\lambda), X_{2}(\lambda)$, and $X_{J}$, given by

$$
X_{1}(\lambda)=\sqrt{1+\lambda r^{2}} \frac{\partial}{\partial x}, \quad X_{2}(\lambda)=\sqrt{1+\lambda r^{2}} \frac{\partial}{\partial y}, \quad X_{J}=x \frac{\partial}{\partial y}-y \frac{\partial}{\partial x},
$$

namely

$$
\begin{aligned}
& X_{1}^{t}(\lambda)=\sqrt{1+\lambda r^{2}} \frac{\partial}{\partial x}+\lambda \frac{x v_{x}+y v_{y}}{\sqrt{1+\lambda r^{2}}} \frac{\partial}{\partial v_{x}}, \quad X_{2}^{t}(\lambda)=\sqrt{1+\lambda r^{2}} \frac{\partial}{\partial y}+\lambda \frac{x v_{x}+y v_{y}}{\sqrt{1+\lambda r^{2}}} \frac{\partial}{\partial v_{y}}, \\
& X_{J}^{t}=x \frac{\partial}{\partial y}-y \frac{\partial}{\partial x}+v_{x} \frac{\partial}{\partial v_{y}}-v_{y} \frac{\partial}{\partial v_{x}},
\end{aligned}
$$

These vector fields close on a Lie algebra:

$$
\left[X_{1}(\lambda), X_{2}(\lambda)\right]=\lambda X_{J}, \quad\left[X_{1}(\lambda), X_{J}\right]=X_{2}(\lambda), \quad\left[X_{2}(\lambda), X_{J}\right]=-X_{1}(\lambda),
$$

which is either isomorphic to $\mathfrak{s o}(3, \mathbb{R})$ when $\lambda>0$, to $\mathfrak{s o}(2,1)$ when $\lambda<0$, or to the Lie algebra of the Euclidean group for $\lambda=0$.

The appropriate generalization for the potential of the nonlinear two-dimensional $\lambda$-dependent oscillator is given by

$$
V_{\lambda}(x, y)=\frac{\alpha^{2}}{2} \frac{x^{2}+y^{2}}{1+\lambda\left(x^{2}+y^{2}\right)} .
$$

This bi-dimensional nonlinear oscillator is completely integrable [25], because one can show that, if $K_{1}$ and $K_{2}$ are the functions

$$
K_{1}=P_{1}(\lambda)+i \alpha \frac{x}{\sqrt{1+\lambda r^{2}}}, \quad K_{2}=P_{2}(\lambda)+i \alpha \frac{y}{\sqrt{1+\lambda r^{2}}},
$$

with

$$
P_{1}(\lambda)=\frac{v_{x}-\lambda J y}{\sqrt{1+\lambda r^{2}}}, \quad P_{2}(\lambda)=\frac{v_{y}+\lambda J x}{\sqrt{1+\lambda r^{2}}}, \quad J=x v_{y}-y v_{x},
$$

then the complex functions $K_{i j}$ defined as $K_{i j}=K_{i} K_{j}^{*}, i, j=1,2$, are constants of motion.

In fact, the time-evolution of the functions $K_{1}$ and $K_{2}$ is

$$
\frac{d}{d t} K_{1}=\frac{i \alpha}{1+\lambda r^{2}} K_{1}, \quad \frac{d}{d t} K_{2}=\frac{i \alpha}{1+\lambda r^{2}} K_{2},
$$

from which we see that the complex functions $K_{i j}$ are constants of the motion.

Therefore the system is super-integrable with the following first integrals of motion:

$$
I_{1}(\lambda)=\left|K_{1}\right|^{2}, \quad I_{2}(\lambda)=\left|K_{2}\right|^{2}, \quad I_{3}=\operatorname{Im}\left(K_{12}\right)=\alpha\left(x v_{y}-y v_{x}\right) .
$$




\subsection{Nonlinear Smorodinsky-Winternitz system}

We can consider a deformed Smorodinsky-Winternitz system with Lagrangian [26]

$$
L=\frac{1}{2\left(1+\lambda r^{2}\right)}\left[v_{x}^{2}+v_{y}^{2}+\lambda\left(x v_{y}-y v_{x}\right)^{2}\right]-\frac{\alpha^{2}}{2} \frac{x^{2}+y^{2}}{1+\lambda\left(x^{2}+y^{2}\right)}+\frac{k_{2}}{x^{2}}+\frac{k_{3}}{y^{2}} .
$$

The E-L equations cannot be directly solved in a simple way, but one can check by direct computation the existence of three independent constants of motion:

$$
\begin{aligned}
& I_{1}=P_{1}^{2}+\frac{\alpha^{2} x^{2}}{1+\lambda\left(x^{2}+y^{2}\right)}+2 k_{2} \frac{1+\lambda y^{2}}{x^{2}}, \\
& I_{2}=P_{2}^{2}+\frac{\alpha^{2} y^{2}}{1+\lambda\left(x^{2}+y^{2}\right)}+2 k_{3} \frac{1+\lambda x^{2}}{y^{2}}, \quad I_{3}=J^{2}+2 k_{2} \frac{y^{2}}{x^{2}}+2 k_{3} \frac{x^{2}}{y^{2}} .
\end{aligned}
$$

This proves that for any value of $\lambda$ this system is completely integrable. Note that for $\lambda=0$ the previous constants of motion reduce to the three first integrals of the S-W potential. Consequently, this system is a deformation of the $\mathrm{S}-\mathrm{W}$ potential which preserves the completely integrability.

\section{Hamilton-Jacobi super-separability of the previous systems}

The Legendre transformation for a Lagrangian $L_{\lambda}=T_{2}(\lambda)-V(x)$ is given by [25]

$$
p_{x}=\frac{\left(1+\lambda y^{2}\right) v_{x}-\lambda x y v_{y}}{1+\lambda r^{2}}, \quad p_{y}=\frac{\left(1+\lambda x^{2}\right) v_{y}-\lambda x y v_{x}}{1+\lambda r^{2}} .
$$

Note that $x p_{y}-y p_{x}=x v_{y}-y v_{x}$.

The general expression for a $\lambda$-dependent Hamiltonian is

$$
H(\lambda)=\frac{1}{2}\left[p_{x}^{2}+p_{y}^{2}+\lambda\left(x p_{x}+y p_{y}\right)^{2}\right]+\frac{1}{2} \alpha^{2} V(x, y),
$$

and hence the associated Hamilton-Jacobi equation is

$$
\left(\frac{\partial S}{\partial x}\right)^{2}+\left(\frac{\partial S}{\partial y}\right)^{2}+\lambda\left(x \frac{\partial S}{\partial x}+y \frac{\partial S}{\partial y}\right)^{2}+\alpha^{2} V(x, y)=2 E
$$

This equation is not separable in $(x, y)$ coordinates but there exist three particular orthogonal coordinate systems, and three particular families of associated potentials, for which such a Hamiltonian admits Hamilton-Jacobi separability:

(i) In terms of the new coordinates $\left(z_{x}, y\right), z_{x}=x / \sqrt{1+\lambda y^{2}}$, the Hamilton-Jacobi equation becomes:

$$
\left(1+\lambda z_{x}^{2}\right)\left(\frac{\partial S}{\partial z_{x}}\right)^{2}+\left(1+\lambda y^{2}\right)^{2}\left(\frac{\partial S}{\partial y}\right)^{2}+\alpha^{2}\left(1+\lambda y^{2}\right) V=2\left(1+\lambda y^{2}\right) E
$$

so if the potential $V(x, y)$ can be written in the form

$$
V=\frac{W_{1}\left(z_{x}\right)}{1+\lambda y^{2}}+W_{2}(y)
$$

then the equation becomes separable.

The potential is therefore integrable with the following two quadratic integrals of motion

$$
I_{1}(\lambda)=\left(1+\lambda r^{2}\right) p_{x}^{2}+\alpha^{2} W_{1}\left(z_{x}\right),
$$




$$
I_{2}(\lambda)=\left(1+\lambda r^{2}\right) p_{y}^{2}-\lambda J^{2}+\alpha^{2}\left(W_{2}(y)-\frac{\lambda y^{2}}{1+\lambda y^{2}} W_{1}\left(z_{x}\right)\right) .
$$

and $H_{\lambda}$ can be written as: $H_{\lambda}=(1 / 2)\left(I_{1}(\lambda)+I_{2}(\lambda)\right)$.

(ii) Similarly, using coordinates $\left(x, z_{y}\right), z_{y}=y / \sqrt{1+\lambda x^{2}}$, the Hamilton-Jacobi equation becomes:

$$
\left(1+\lambda x^{2}\right)^{2}\left(\frac{\partial S}{\partial x}\right)^{2}+\left(1+\lambda z_{y}^{2}\right)\left(\frac{\partial S}{\partial z_{y}}\right)^{2}+\alpha^{2}\left(1+\lambda x^{2}\right) V=2\left(1+\lambda x^{2}\right) E
$$

therefore, if the potential $V(x, y)$ can be written on the form

$$
V=W_{1}(x)+\frac{W_{2}\left(z_{y}\right)}{1+\lambda x^{2}}
$$

then the equation becomes separable.

The potential is therefore integrable with the following two quadratic integrals of motion

$$
\begin{aligned}
& I_{1}(\lambda)=\left(1+\lambda r^{2}\right) p_{x}^{2}-\lambda J^{2}+\alpha^{2}\left(W_{1}(x)-\frac{\lambda x^{2}}{1+\lambda x^{2}} W_{1}\left(z_{y}\right)\right), \\
& I_{2}(\lambda)=\left(1+\lambda r^{2}\right) p_{y}^{2}+\alpha^{2} W_{2}\left(z_{y}\right)
\end{aligned}
$$

and $H_{\lambda}$ can be written as: $H_{\lambda}=(1 / 2)\left(I_{1}(\lambda)+I_{2}(\lambda)\right)$.

(iii) In polar coordinates $(r, \phi)$ the Hamiltonian $H(\lambda)$ is

$$
H(\lambda)=\frac{1}{2}\left[\left(1+\lambda r^{2}\right) p_{r}^{2}+\frac{p_{\phi}^{2}}{r^{2}}\right]+\frac{\alpha^{2}}{2} V(r, \phi)
$$

so that the Hamilton-Jacobi equation is given by

$$
\left(1+\lambda r^{2}\right)\left(\frac{\partial S}{\partial r}\right)^{2}+\frac{1}{r^{2}}\left(\frac{\partial S}{\partial \phi}\right)^{2}+\alpha^{2} V(r, \phi)=2 E
$$

If the potential $V$ is of the form

$$
V=F(r)+\frac{G(\phi)}{r^{2}}
$$

then the equation admits separability

$$
r^{2}\left(1+\lambda r^{2}\right)\left(\frac{\partial S}{\partial r}\right)^{2}+r^{2}\left(\alpha^{2} F(r)-2 E\right)+\left(\frac{\partial S}{\partial \phi}\right)^{2}+\alpha^{2} G(\phi)=0
$$

The potential $V$ is integrable with the following two quadratic integrals of motion:

$$
\begin{aligned}
& I_{1}(\lambda)=\left(1+\lambda r^{2}\right) p_{r}^{2}+\frac{1-r^{2}}{r^{2}} p_{\phi}^{2}+\alpha^{2}\left[F(r)+\frac{1-r^{2}}{r^{2}} G(\phi)\right], \\
& I_{2}(\lambda)=p_{\phi}^{2}+\alpha^{2} G(\phi)
\end{aligned}
$$

and $H_{\lambda}$ can be written as: $H_{\lambda}=(1 / 2)\left(I_{1}(\lambda)+I_{2}(\lambda)\right)$. 


\subsection{Super-separability of the nonlinear oscillator}

Using the previous results one can see that the potential

$$
V_{\lambda}=\frac{\alpha^{2}}{2} \frac{x^{2}+y^{2}}{1+\lambda\left(x^{2}+y^{2}\right)}
$$

is actually super-separable (separable in more than one system of coordinates) [25]. In fact, the potential for the deformed oscillator we are considering can be alternatively written as follows:

$$
V_{\lambda}=\frac{\alpha^{2}}{2} \frac{1}{1+\lambda y^{2}}\left[\frac{z_{x}^{2}}{1+\lambda z_{x}^{2}}+y^{2}\right]=\frac{\alpha^{2}}{2} \frac{1}{1+\lambda x^{2}}\left[x^{2}+\frac{z_{y}^{2}}{1+\lambda z_{y}^{2}}\right]=\frac{\alpha^{2}}{2} \frac{r^{2}}{1+\lambda r^{2}} .
$$

Consequently, the Hamiltonian

$$
H(\lambda)=\frac{1}{2}\left[p_{x}^{2}+p_{y}^{2}+\lambda\left(x p_{x}+y p_{y}\right)^{2}\right]+\frac{\alpha^{2}}{2} \frac{x^{2}+y^{2}}{1+\lambda\left(x^{2}+y^{2}\right)}
$$

admits the following decomposition

$$
H(\lambda)=H_{1}(\lambda)+H_{2}(\lambda)-\lambda H_{3}
$$

where the three partial functions $H_{1}, H_{2}$, and $H_{3}$ are:

$$
\begin{aligned}
& H_{1}(\lambda)=\frac{1}{2}\left[\left(1+\lambda r^{2}\right) p_{x}^{2}+\alpha^{2} \frac{x^{2}}{1+\lambda r^{2}}\right], \\
& H_{2}(\lambda)=\frac{1}{2}\left[\left(1+\lambda r^{2}\right) p_{y}^{2}+\alpha^{2} \frac{y^{2}}{1+\lambda r^{2}}\right], \quad H_{3}=\frac{1}{2}\left(x p_{y}-y p_{x}\right)^{2},
\end{aligned}
$$

and each one has a vanishing Poisson bracket with $H$,

$$
\left\{H, H_{1}(\lambda)\right\}=0, \quad\left\{H, H_{2}(\lambda)\right\}=0, \quad\left\{H, H_{3}(\lambda)\right\}=0 .
$$

\subsection{Super-separability of the deformed S-W system}

The important point is that, in a similar manner, the $\lambda$-deformed Smorodinsky-Winternitz potential

$$
V_{\lambda, k}=\frac{\alpha^{2}}{2}\left(\frac{x^{2}+y^{2}}{1+\lambda\left(x^{2}+y^{2}\right)}\right)+\frac{k_{2}}{x^{2}}+\frac{k_{3}}{y^{2}}
$$

can be alternatively written in the following three different ways [26]

$$
\begin{aligned}
V_{\lambda, k} & =\frac{\alpha^{2}}{2}\left(\frac{1}{1+\lambda y^{2}}\right)\left[\frac{z_{x}^{2}}{1+\lambda z_{x}^{2}}+y^{2}\right]+\left(\frac{k_{2}}{1+\lambda y^{2}}\right) \frac{1}{z_{x}^{2}}+\frac{k_{3}}{y^{2}} \\
& =\frac{\alpha^{2}}{2}\left(\frac{1}{1+\lambda x^{2}}\right)\left[x^{2}+\frac{z_{y}^{2}}{1+\lambda z_{y}^{2}}\right]+\frac{k_{2}}{x^{2}}+\left(\frac{k_{3}}{1+\lambda x^{2}}\right) \frac{1}{z_{y}^{2}} \\
& =\frac{\alpha^{2}}{2}\left(\frac{r^{2}}{1+\lambda r^{2}}\right)+\frac{k_{2}}{r^{2} \cos ^{2} \phi}+\frac{k_{3}}{r^{2} \sin ^{2} \phi} .
\end{aligned}
$$

Therefore, it is super-separable since it is separable in three different systems of coordinates, $\left(z_{x}, y\right),\left(x, z_{y}\right)$, and $(r, \phi)$. This remarkable property means that the Hamiltonian

$$
H_{\lambda, k}=\frac{1}{2}\left[p_{x}^{2}+p_{y}^{2}+\lambda\left(x p_{x}+y p_{y}\right)^{2}\right]+V_{\lambda, k}
$$


admits the following decomposition $H_{\lambda, k}=H_{p_{x}}+H_{p_{y}}-\lambda H_{J}$, where the three partial functions $H_{1}, H_{2}$, and $H_{3}$, are given by

$$
\begin{aligned}
& H_{p_{x}}=\frac{1}{2}\left[\left(1+\lambda r^{2}\right) p_{x}^{2}+\alpha^{2}\left(\frac{x^{2}}{1+\lambda r^{2}}\right)\right]+k_{2}\left(\frac{1+\lambda y^{2}}{x^{2}}\right), \\
& H_{p_{y}}=\frac{1}{2}\left[\left(1+\lambda r^{2}\right) p_{y}^{2}+\alpha^{2}\left(\frac{y^{2}}{1+\lambda r^{2}}\right)\right]+k_{3}\left(\frac{1+\lambda x^{2}}{y^{2}}\right), \\
& H_{J}=\frac{1}{2}\left(x p_{y}-y p_{x}\right)^{2}+k_{2} \frac{y^{2}}{x^{2}}+k_{3} \frac{x^{2}}{y^{2}} .
\end{aligned}
$$

Each one of these three terms has a vanishing Poisson bracket with $H$ for any value of the parameters $\lambda, k_{2}$ and $k_{3}$

$$
\left\{H_{\lambda, k}, H_{p_{x}}\right\}=0, \quad\left\{H_{\lambda, k}, H_{p_{y}}\right\}=0, \quad\left\{H_{\lambda, k}, H_{J}\right\}=0 .
$$

Consequently, the Hamiltonian can be written as a sum, not of two, but of three integrals of motion. The third one represents the contribution of the angular momentum $J$ to $H$, with the parameter $\lambda$ as a coefficient, therefore vanishing in the limit $\lambda \rightarrow 0$.

\section{A geometric interpretation}

The existence of additional constants of motion for the harmonic oscillator in a spherical geometry was studied by Higgs in 1979 [36]. The Higgs approach considers the motion on $S^{n}$, embedded in the Euclidean space $\mathbb{E}^{n+1}$, by means of a central (also known as gnomonic) projection on a plane $\Pi^{n}$ tangent to $S^{n}$ at a chosen point. In this way he could study the properties of the spherical version of the Fradkin tensor. A different alternative approach is discussed in $[17,20]$ by using curvature-dependent trigonometric and hyperbolic functions, where use is made of the curvature $\kappa$ as a parameter so that the dynamics can be studied at the same time in the sphere $S^{2}$ and in the hyperbolic plane $H^{2}$.

In differential geometric terms, the three spaces with constant curvature, sphere $S^{2}$, Euclidean plane $\mathbb{E}^{2}$, and hyperbolic plane $H^{2}$, can be considered as three different situations inside a family of Riemannian manifolds $M_{\kappa}^{2}=\left(S_{\kappa}^{2}, \mathbb{E}^{2}, H_{\kappa}^{2}\right)$ with the curvature $\kappa$ as a parameter $\kappa \in \mathbb{R}$. In order to obtain mathematical expressions valid for all the values of $\kappa$, it is convenient to make use of the following $\kappa$-trigonometric functions

$$
\mathrm{C}_{\kappa}(x)=\left\{\begin{array}{ll}
\cos \sqrt{\kappa} x & \text { if } \quad \kappa>0, \\
1 & \text { if } \quad \kappa=0, \\
\cosh \sqrt{-\kappa} x & \text { if } \kappa<0,
\end{array} \quad \mathrm{~S}_{\kappa}(x)= \begin{cases}\frac{1}{\sqrt{\kappa}} \sin \sqrt{\kappa} x & \text { if } \kappa>0, \\
x & \text { if } \kappa=0, \\
\frac{1}{\sqrt{-\kappa}} \sinh \sqrt{-\kappa} x & \text { if } \quad \kappa<0,\end{cases}\right.
$$

and the $\kappa$-dependent tangent function $\mathrm{T}_{\kappa}(x)$ defined in the natural way, $\mathrm{T}_{\kappa}(x)=\mathrm{S}_{\kappa}(x) / \mathrm{C}_{\kappa}(x)$. The fundamental properties of these curvature-dependent trigonometric functions are

$$
\mathrm{C}_{\kappa}^{2}(x)+\kappa \mathrm{S}_{\kappa}^{2}(x)=1
$$

and

$$
\begin{aligned}
& \mathrm{C}_{\kappa}(2 x)=\mathrm{C}_{\kappa}^{2}(x)-\kappa \mathrm{S}_{\kappa}^{2}(x), \quad \frac{d}{d x} \mathrm{~S}_{\kappa}(x)=\mathrm{C}_{\kappa}(x), \\
& \mathrm{S}_{\kappa}(2 x)=2 \mathrm{~S}_{\kappa}(x) \mathrm{C}_{\kappa}(x), \quad \frac{d}{d x} \mathrm{C}_{\kappa}(x)=-\kappa \mathrm{S}_{\kappa}(x) .
\end{aligned}
$$


If in the Lagrangian of the nonlinear oscillator in one dimension

$$
L_{\lambda}(x, \dot{x})=\frac{1}{2} \frac{1}{1+\lambda x^{2}}\left(\dot{x}^{2}-\alpha^{2} x^{2}\right),
$$

we consider the change of variable $x=\mathrm{S}_{\kappa}(u)$, where $\lambda=-\kappa$, we find that

$$
1+\lambda x^{2}=1-\kappa x^{2}=1-\kappa \mathrm{S}_{\kappa}^{2}(u)=\mathrm{C}_{\kappa}^{2}(u)
$$

and as $d x / d u=\mathrm{C}_{\kappa}(u)$, we see that

$$
\dot{x}=\frac{d x}{d u} \dot{u}=\mathrm{C}_{\kappa}(u) \dot{u}
$$

and therefore the Lagrangian becomes

$$
L_{\kappa}(u, \dot{u})=\frac{1}{2} \frac{\mathrm{C}_{\kappa}^{2}(u) \dot{u}^{2}}{\mathrm{C}_{\kappa}^{2}(u)}-\frac{1}{2} \frac{\alpha^{2} \mathrm{~S}_{\kappa}^{2}(u)}{\mathrm{C}_{\kappa}^{2}(u)}=\frac{1}{2} \dot{u}^{2}-\frac{\alpha^{2}}{2} \mathrm{~T}_{\kappa}^{2}(u),
$$

the vector field $X_{x}$ turns out to be

$$
X=\mathrm{C}_{\kappa}(u) \frac{d u}{d x} \frac{\partial}{\partial u}=\frac{\partial}{\partial u},
$$

and the metric giving rise to the Lagrangian $g=d u \otimes d u$. We note that the expression $\mathrm{T}_{\kappa}^{2}(u)$ of the above one-dimensional potential appears as directly related with the potential of the two-dimensional harmonic oscillator on a space with curvature $\kappa$.

The expression of the differential element of distance in geodesic polar coordinates $(\rho, \phi)$ on the family $M_{\kappa}^{2}=\left(S_{\kappa}^{2}, \mathbb{E}^{2}, H_{\kappa}^{2}\right)$, can be written as follows $d s_{\kappa}^{2}=d \rho^{2}+\mathrm{S}_{\kappa}^{2}(\rho) d \phi^{2}$, so that it reduces to

$$
d s_{1}^{2}=d \rho^{2}+\left(\sin ^{2} \rho\right) d \phi^{2}, \quad d s_{0}^{2}=d \rho^{2}+\rho^{2} d \phi^{2}, \quad d s_{-1}^{2}=d \rho^{2}+\left(\sinh ^{2} \rho\right) d \phi^{2},
$$

in the three particular cases $\kappa=1,0,-1$ of the unit sphere, Euclidean plane, and 'unit' Lobachewski plane, respectively. Note that $\rho$ denotes the distance along a geodesic on the manifold $M_{\kappa}^{2}$; for example, in the spherical $\kappa>0$ case, $\rho$ is the distance of the point to the origin (e.g., the North pole) along a maximum circle.

Therefore, the Lagrangian for the geodesic (free) motion on the spaces $\left(S_{\kappa}^{2}, \mathbb{E}^{2}, H_{\kappa}^{2}\right)$ is

$$
L_{\kappa}\left(\rho, \phi, v_{\rho}, v_{\phi}\right)=T_{\kappa}\left(\rho, \phi, v_{\rho}, v_{\phi}\right)=\frac{1}{2}\left(v_{\rho}^{2}+\mathrm{S}_{\kappa}^{2}(\rho) v_{\phi}^{2}\right),
$$

and the Lagrangian for a general mechanical system (Riemannian metric minus a potential) is

$$
L_{\kappa}\left(\rho, \phi, v_{\rho}, v_{\phi}\right)=\frac{1}{2}\left(v_{\rho}^{2}+\mathrm{S}_{\kappa}^{2}(\rho) v_{\phi}^{2}\right)-U(\rho, \phi, \kappa) .
$$

The spherical and hyperbolic harmonic oscillators are characterized by the following Lagrangians with curvature $\kappa[17,20]$

$$
L_{\kappa}\left(\rho, \phi, v_{\rho}, v_{\phi}\right)=\frac{1}{2}\left(v_{\rho}^{2}+\mathrm{S}_{\kappa}^{2}(\rho) v_{\phi}^{2}\right)-\frac{1}{2} \omega_{0}^{2} \mathrm{~T}_{\kappa}^{2}(\rho),
$$

i.e. the harmonic oscillators on the unit sphere (Higgs oscillator), on the Euclidean plane, or on the unit Lobachewski plane, are:

$$
U_{1}(\rho)=\frac{1}{2} \omega_{0}^{2} \tan ^{2} \rho, \quad U_{0}(\rho)=\frac{1}{2} \omega_{0}^{2} \rho^{2}, \quad U_{-1}(\rho)=\frac{1}{2} \omega_{0}^{2} \tanh ^{2} \rho .
$$


Next we study the behavior of $L_{\kappa}$ under two different changes of variables.

1 . If we consider the $\kappa$-dependent change $(\rho, \phi) \rightarrow\left(r^{\prime}, \phi\right)$ given by $r^{\prime}=\mathrm{T}_{\kappa}(\rho)$, then the Lagrangian $L_{\kappa}$ becomes

$$
L_{H \kappa}\left(r^{\prime}, \phi, v_{r^{\prime}}, v_{\phi}\right)=\frac{1}{2}\left(\frac{v_{r^{\prime}}{ }^{2}}{\left(1+\kappa r^{\prime 2}\right)^{2}}+\frac{{r^{\prime}}^{2} v_{\phi}^{2}}{\left(1+\kappa r^{\prime 2}\right)}\right)-\frac{1}{2} \alpha^{2} r^{\prime 2} .
$$

This function coincides, in the spherical $\kappa>0$ case, with the Lagrangian studied by Higgs in [36]. In Cartesian coordinates $(x, y)$ it reduces to

$$
L_{H \kappa}\left(x, y, v_{x}, v_{y}\right)=\frac{1}{2} \frac{1}{\left(1+\kappa r^{\prime 2}\right)}\left[v_{x}^{2}+v_{y}^{2}+\kappa\left(x v_{y}-y v_{x}\right)^{2}\right]-\frac{1}{2} \alpha^{2} r^{\prime 2}, \quad r^{\prime 2}=x^{2}+y^{2} .
$$

2. Let us consider the $\kappa$-dependent change $(\rho, \phi) \rightarrow(r, \phi)$ given by $r=\mathrm{S}_{\kappa}(\rho), \lambda=-\kappa$. Then the Lagrangian $L_{\kappa}$ becomes

$$
L_{\lambda}\left(r, \phi, v_{r}, v_{\phi}\right)=\frac{1}{2}\left(\frac{v_{r}^{2}}{1+\lambda r^{2}}+r^{2} v_{\phi}^{2}\right)-\frac{\alpha^{2}}{2}\left(\frac{r^{2}}{1+\lambda r^{2}}\right) .
$$

Therefore, if we change to Cartesian coordinates $(x, y)$ we arrive to

$$
\begin{aligned}
& L_{\lambda}\left(x, y, v_{x}, v_{y}\right)=\frac{1}{2}\left(\frac{1}{1+\lambda r^{2}}\right)\left[v_{x}^{2}+v_{y}^{2}+\lambda\left(x v_{y}-y v_{x}\right)^{2}\right]-\frac{\alpha^{2}}{2}\left(\frac{r^{2}}{1+\lambda r^{2}}\right), \\
& r^{2}=x^{2}+y^{2} .
\end{aligned}
$$

This function is just the Lagrangian obtained in [25] as the natural generalization of the onedimensional Lagrangian $L_{\lambda}\left(x, v_{x}\right)$ for the nonlinear equation (1) of Mathews and Lakshmanan.

We thus have three different and alternative ways of describing the harmonic oscillator on spaces of constant curvature: the original $\kappa$-dependent trigonometric (hyperbolic) Lagrangian $L_{\kappa}$ and the two other approaches, $L_{H \kappa}$ and $L_{\lambda}$, obtained from it.

The Higgs approach [36] has been studied by many authors (see e.g. [37, 38] and references therein) mainly in relation with the theory of dynamical symmetries. Concerning the $\lambda$-dependent Lagrangian $L_{\lambda}$, it has similarities with $L_{H \kappa}$ but it does not coincide with it. In the model of Higgs $\kappa$ (or $\lambda$ ) is present in the kinetic term $T$ in a different way and the potential $V$ appears as $\kappa$-independent; this affects to the Hamiltonian formalism. On the other side each one of these three formalisms can be used for study of the $\kappa$-dependent version of the S-W system. In the language of $L_{\kappa}$ the potential, that was studied in [13, 20], is given by

$$
\begin{aligned}
& U_{a}(r, \phi, \kappa)=k_{1} U_{a}^{1}+k_{2} U_{a}^{2}+k_{3} U_{a}^{3}+k_{0}, \quad k_{1}=\frac{1}{2} \omega_{0}^{2}, \\
& U_{a}^{1}=\mathrm{T}_{\kappa}^{2}(\rho), \quad U_{a}^{2}=\frac{1}{\left(\mathrm{~S}_{\kappa}(\rho) \cos \phi\right)^{2}}, \quad U_{a}^{3}=\frac{1}{\left(\mathrm{~S}_{\kappa}(\rho) \sin \phi\right)^{2}},
\end{aligned}
$$

with integrals of motion given by

$$
\begin{aligned}
& I_{1}(\kappa)=P_{1}^{2}(\kappa)+\omega_{0}^{2}\left(\mathrm{~T}_{\kappa}(\rho) \cos \phi\right)^{2}+\frac{2 k_{2}}{\left(\mathrm{~T}_{\kappa}(\rho) \cos \phi\right)^{2}}, \\
& I_{2}(\kappa)=P_{2}^{2}(\kappa)+\omega_{0}^{2}\left(\mathrm{~T}_{\kappa}(\rho) \sin \phi\right)^{2}+\frac{2 k_{3}}{\left(\mathrm{~T}_{\kappa}(\rho) \sin \phi\right)^{2}}, \\
& I_{3}(\kappa)=J^{2}(\kappa)+\frac{2 k_{2}}{\cos ^{2} \phi}+\frac{2 k_{3}}{\sin ^{2} \phi},
\end{aligned}
$$


with $P_{1}(\kappa), P_{2}(\kappa)$ and $J(\kappa)$ given by

$$
\begin{aligned}
& P_{1}(\kappa)=(\cos \phi) v_{\rho}-\left(\mathrm{C}_{\kappa}(\rho) \mathrm{S}_{\kappa}(\rho) \sin \phi\right) v_{\phi}, \\
& P_{2}(\kappa)=(\sin \phi) v_{\rho}+\left(\mathrm{C}_{\kappa}(\rho) \mathrm{S}_{\kappa}(\rho) \cos \phi\right) v_{\phi}, \quad J(\kappa)=\mathrm{S}_{\kappa}^{2}(\rho) v_{\phi} .
\end{aligned}
$$

This S-W system has been studied, using a different approach, in the two- and three- dimensional sphere in [10], in the two-dimensional hyperboloid in [12] and in the complex two-sphere in [16].

One of the advantages of the $L_{\lambda}$ approach is that the Euler-Lagrange equations can be directly solved and the general solution has a rather simple form that can be interpreted as "quasiharmonic" nonlinear oscillations [25]; other important advantage is that it is very appropriate for the study of the quantum oscillator.

In what follows we will focus our attention on the quantum Hamiltonian dynamics determined by the $\lambda$-dependent Lagrangian $L_{\lambda}$.

\section{The one-dimensional quantum nonlinear oscillator}

Let us consider the quantum case for $n=1$. The problem is to define the quantum operator defining the Hamiltonian of this position-dependent mass system, because the mass and the momentum $P$ do not commute and this fact gives rise to an ambiguity in the ordering of factors.

Instead of using traditional procedures as Weyl ordering we shall develop an alternative method of quantization [39]. We first remark that the vector field

$$
X_{x}(\lambda)=\sqrt{1+\lambda x^{2}} \frac{\partial}{\partial x}
$$

which was a Killing vector for the metric corresponding to the kinetic energy

$$
g=\left(1+\lambda x^{2}\right)^{-1} d x \otimes d x
$$

and generates the translations in this Riemann space, does not leave invariant the natural measure in the real line but the only invariant measures are the multiples of

$$
d \mu=\left(1+\lambda x^{2}\right)^{-1 / 2} d x .
$$

This suggests us to consider the Hilbert space $\mathcal{L}^{2}(\mathbb{R}, d \mu)$ and the remarkable fact is that the adjoint of the differential operator $\sqrt{1+\lambda x^{2}} \partial / \partial x$ in such space is the opposite of such operator.

The Legendre transformation corresponding to this deformed kinetic energy is defined by

$$
p=\frac{v_{x}}{1+\lambda x^{2}}
$$

and then the Hamiltonian function for the free particle is given by

$$
H=\left(1+\lambda x^{2}\right) \frac{p^{2}}{2}=\frac{1}{2}\left(\sqrt{1+\lambda x^{2}} p\right)^{2} .
$$

The usual prescription of canonical quantization does not present any ambiguity because the linear operator (we put $\hbar=1$ )

$$
\widehat{P}=-i \sqrt{1+\lambda x^{2}} \frac{\partial}{\partial x}
$$

is self-adjoint in the space $\mathcal{L}^{2}(\mathbb{R}, d \mu)$. Note that with the above mentioned change of coordinates, $x=\mathrm{S}_{\kappa}(u), d \mu=d u$ and

$$
\frac{\partial}{\partial u}=\mathrm{C}_{\kappa}(u) \frac{\partial}{\partial x}=\sqrt{1-\kappa \mathrm{S}_{\kappa}^{2}(u)} \frac{\partial}{\partial x}=\sqrt{1+\lambda x^{2}} \frac{\partial}{\partial x}
$$

is self-adjoint in the space $\mathcal{L}^{2}(\mathbb{R}, d u)$, and then $\widehat{P}$ generates translations in these coordinates $u$. 
The quantum Hamiltonian operator of the free particle is

$$
\widehat{H}=-\frac{1}{2}\left(\sqrt{1+\lambda x^{2}} \frac{\partial}{\partial x}\right)^{2}=-\frac{1}{2}\left(1+\lambda x^{2}\right) \frac{\partial^{2}}{\partial x^{2}}-\frac{1}{2} \lambda x \frac{\partial}{\partial x}
$$

and in presence of an interaction $V_{1}(x)$ the Hamiltonian will be

$$
\widehat{H}_{1}=-\frac{1}{2}\left(1+\lambda x^{2}\right) \frac{d^{2}}{d x^{2}}-\frac{1}{2} \lambda x \frac{d}{d x}+V_{1}(x) .
$$

We are interested in the case of the nonlinear oscillator for which

$$
\widehat{H}_{1}=-\frac{1}{2}\left(1+\lambda x^{2}\right) \frac{d^{2}}{d x^{2}}-\frac{1}{2} \lambda x \frac{d}{d x}+\frac{1}{2} \frac{\alpha^{2} x^{2}}{1+\lambda x^{2}} .
$$

If $m$ and $\hbar$ are taken into account, and with the change of parameter, to be more clear later, given by

$$
\alpha^{2}=\beta\left(\beta+\frac{\hbar}{m} \lambda\right)
$$

it is enough to define the dimensionless variables

$$
y=\sqrt{\frac{m \beta}{\hbar}}, \quad \Lambda=\frac{\hbar}{m \beta} \lambda
$$

and then $\widehat{H}_{1}$ is written as

$$
\widehat{H}_{1}=\beta \hbar\left[-\frac{1}{2}\left(1+\Lambda y^{2}\right) \frac{d^{2}}{d y^{2}}-\frac{1}{2} \Lambda y \frac{d}{d y}+\frac{1}{2}(1+\Lambda) \frac{y^{2}}{1+\lambda y^{2}}\right] .
$$

The time-independent Schrödinger equation

$$
\widehat{H}_{1} \Psi=E \Psi, \quad E=\hbar \beta \mathcal{E}
$$

becomes

$$
\left[-\frac{1}{2}\left(1+\Lambda y^{2}\right) \frac{d^{2}}{d y^{2}}-\frac{1}{2} \Lambda y \frac{d}{d y}+\frac{1}{2}(1+\Lambda) \frac{y^{2}}{1+\lambda y^{2}}\right] \Psi=\mathcal{E} \Psi .
$$

With the change of variable [40]

$$
\Psi(y, \Lambda)=\varphi(y, \Lambda)\left(1+\Lambda y^{2}\right)^{-1 /(2 \Lambda)}
$$

the eigenvalue equation becomes

$$
\left(1+\Lambda y^{2}\right) \varphi^{\prime \prime}+(\Lambda-2) y \varphi^{\prime}+(2 \mathcal{E}-1) \varphi=0
$$

and assuming a power series development

$$
\varphi(y)=\sum_{n \geq 0} a_{n} y^{n}
$$

the following recursion relation for the coefficients is obtained:

$$
a_{n+2}=(-1)^{n} \frac{a_{n}}{(n+2)(n+1)}[n(\Lambda n-2+(2 \mathcal{E}-1)] .
$$


Therefore the general solution is determined by the values of the coefficients $a_{0}$ and $a_{1}$. In particular we can write the solutions $y_{0}$ and $y_{1}$ determined by $a_{0}=1, a_{1}=0$ and $a_{0}=1, a_{1}=0$, respectively. The solution $y_{0}$ is an even function while the $y_{1}$ function is odd. The general solution will be written as $y=a_{0} y_{0}+a_{1} y_{1}$.

The convergence radius of such power series is

$$
R=\frac{1}{\sqrt{|\Lambda|}} \quad \text { because } \quad \lim _{n \rightarrow \infty}\left|\frac{a_{n+2}}{a_{n}}\right|=|\Lambda| .
$$

This series $y=a_{0} y_{0}+a_{1} y_{1}$ reduces to a polynomial of degree $p$ when one of the two coefficients vanish and that means that there exists a positive integer number $p$ such that $2 \mathcal{E}-1=2 p-\Lambda p^{2}$, and then

$$
\mathcal{E}_{p}=p\left(1-\Lambda \frac{p}{2}\right)+\frac{1}{2}
$$

The polynomial solutions can be easily found and they have a form quite similar to the corresponding Hermite polynomials.

\section{Factorization method and shape-invariance}

The spectrum of the harmonic oscillator can be found using the factorization method we shall describe in this section [39]. Actually the Hamiltonian can be factorized and we arrive to a shapeinvariant Hamiltonian for which the full point-spectrum can be found by algebraic methods. We take $\hbar=\omega=1$. The eigenvalue problem is (up to a factor $1 / 2$ )

$$
H \psi_{n}=\left(-\frac{d^{2}}{d x^{2}}+x^{2}\right) \psi_{n}=(2 n+1) \psi_{n}
$$

where the Hamiltonian $H$ is such that $H-1=a^{\dagger} a$, with $a=(d / d x+x), a^{\dagger}=(-d / d x+x)$, and then the ground state is found from

$$
a \psi_{0}=\left(\frac{d}{d x}+x\right) \psi_{0}=0
$$

i.e. $\psi_{0} \propto e^{-x^{2} / 2}$. The other eigenstates can be found by applying an iterative way the creation operator $a^{\dagger}$ to the ground state:

$$
\psi_{n}=\frac{1}{\sqrt{2^{n} n !}} a^{\dagger n} \psi_{0} \propto H_{n}(x) e^{-x^{2} / 2},
$$

with $H_{n}(x)$ being the Hermite polynomials.

A similar procedure can be used for the quantum nonlinear oscillator case. We should look for a function $W(x)$, to be called super-potential, in such a way that the operator $A$ and its adjoint operator $A^{+}$, given by

$$
A=\frac{1}{\sqrt{2}}\left(\sqrt{1+\lambda x^{2}} \frac{d}{d x}+W(x)\right), \quad A^{+}=\frac{1}{\sqrt{2}}\left(-\sqrt{1+\lambda x^{2}} \frac{d}{d x}+W(x)\right),
$$

are such that $\widehat{H}_{1}=A^{+} A$, i.e.

$$
\widehat{H}_{1}=A^{+} A=\frac{1}{2}\left[-\sqrt{1+\lambda x^{2}} \frac{d}{d x}+W(x)\right]\left[\sqrt{1+\lambda x^{2}} \frac{d}{d x}+W(x)\right] .
$$


Therefore, in order to the Hamiltonian so found be that of the deformed quadratic energy term together with a potential $V_{1}$, the super-potential function $W$ must satisfy

$$
\sqrt{1+\lambda x^{2}} W^{\prime}-W^{2}+2 V_{1}=0 .
$$

We can define a new quantum Hamiltonian operator

$$
\widehat{H}_{2}=A A^{+}=\left[\sqrt{1+\lambda x^{2}} \frac{d}{d x}+W(x)\right]\left[-\sqrt{1+\lambda x^{2}} \frac{d}{d x}+W(x)\right]
$$

which is called the partner Hamiltonian. The new potential $V_{2}$ is given in terms of $W$ by

$$
V_{2}=\frac{1}{2}\left(\sqrt{1+\lambda x^{2}} W^{\prime}+g W^{2}\right) .
$$

The important fact is that

$$
A \widehat{H}_{1}=\widehat{H}_{2} A, \quad A^{+} \widehat{H}_{2}=\widehat{H}_{1} A^{+} .
$$

When $\widehat{H}_{1}|\Psi\rangle=E|\Psi\rangle$, then, $\widehat{H}_{2} A|\Psi\rangle=A \widehat{H}_{1}|\Psi\rangle=E A|\Psi\rangle$. If $A|\Psi\rangle \neq 0, A|\Psi\rangle$ is an eigenvector of $\widehat{H}_{2}$ corresponding to the same eigenvalue $E$, and similarly, if $|\Phi\rangle$ is an eigenvector of $\widehat{H}_{2}$ with eigenvalue $E$ and such that $A^{+}|\Phi\rangle \neq 0$, then $A^{+}|\Phi\rangle$ is an eigenvector of $\widehat{H}_{1}$ corresponding to the same eigenvalue $E$.

The spectra of $\widehat{H}_{1}$ and $\widehat{H}_{2}$ are then almost identical, the only differences are when either $|\Psi\rangle$ is an eigenvector of $\widehat{H}_{1}$ but $A|\Psi\rangle \neq 0$, or $|\Phi\rangle$ is an eigenvector of $\widehat{H}_{2}$ for which $A^{+}|\Phi\rangle=0$.

Some parameters may appear in the expression of $V_{1}$, and the super-potential function $W$ will also depend on them. The most important case is when the explicit forms of the potential and its partner are quite similar and only differ in the values of the parameters. In this case we say that the problem has shape invariance (see e.g. [41]).

Suppose that a quantum Hamiltonian $\widehat{H}_{1}(\alpha)$ admits a factorization $\widehat{H}_{1}(\alpha)=A^{+}(\alpha) A(\alpha)$ in such a way that the partner Hamiltonian $\widehat{H}_{2}(\alpha)$ is of the same form as $\widehat{H}_{1}(\alpha)$ but for a different value of the parameter $\alpha$. More specifically, there exists a function $f$ such that

$$
\widehat{H}_{2}(\alpha)=\widehat{H}_{1}\left(\alpha_{1}\right)+R\left(\alpha_{1}\right),
$$

where $\alpha_{1}=f(\alpha)$ and $R(\alpha)$ is a constant depending on the parameter $\alpha$. In this case Gendenshteïn developed a method for exact computing of all the spectrum of $\widehat{H}_{1}[41,42,43]$. First, the bound state $\left|\Psi_{0}\right\rangle$ is found by solving $A(\alpha)\left|\Psi_{0}(\alpha)\right\rangle=0$, and has a zero energy. Then, $\left|\Psi_{0}\left(\alpha_{1}\right)\right\rangle$ is an eigenstate of $\widehat{H}_{2}(\alpha)$ with $E_{1}=R\left(\alpha_{1}\right)$, because

$$
\widehat{H}_{2}(\alpha)\left|\Psi_{0}\left(\alpha_{1}\right)\right\rangle=\left(\widehat{H}_{1}\left(\alpha_{1}\right)+R\left(\alpha_{1}\right)\right)\left|\Psi_{0}\left(\alpha_{1}\right)\right\rangle=R\left(\alpha_{1}\right)\left|\Psi_{0}\left(\alpha_{1}\right)\right\rangle,
$$

and $A^{\dagger}(\alpha)\left|\Psi_{0}\left(\alpha_{1}\right)\right\rangle$ is the first excited state of $\widehat{H}_{1}(\alpha)$, with energy $E_{1}=R\left(\alpha_{1}\right)$, because:

$$
\widehat{H}_{1}(\alpha) A^{\dagger}(\alpha)\left|\Psi_{0}\left(\alpha_{1}\right)\right\rangle=A^{\dagger}(\alpha)\left(\widehat{H}_{1}\left(\alpha_{1}\right)+R\left(\alpha_{1}\right)\right)\left|\Psi_{0}\left(\alpha_{1}\right)\right\rangle=R\left(\alpha_{1}\right) A^{\dagger}(\alpha)\left|\Psi_{0}\left(\alpha_{1}\right)\right\rangle .
$$

Iterating the process we find the sequence of energies for $\widehat{H}_{1}(\alpha)$

$$
E_{k}=\sum_{j=1}^{k} R\left(\alpha_{j}\right), \quad E_{0}=0,
$$

the corresponding eigenfunctions being

$$
\left|\Psi_{n}\left(x, \alpha_{0}\right)\right\rangle=A^{\dagger}\left(\alpha_{0}\right) A^{\dagger}\left(\alpha_{1}\right) \cdots A^{\dagger}\left(\alpha_{n-1}\right)\left|\Psi_{0}\left(x, \alpha_{n}\right)\right\rangle,
$$

where $\alpha_{0}=\alpha$ and $\alpha_{j+1}=f\left(\alpha_{j}\right)$, namely, $\alpha_{k}=f^{k}\left(\alpha_{0}\right)=f^{k}(\alpha)$. 
Coming back to the nonlinear oscillator case, if $\beta \in \mathbb{R}$, we define the linear operator in $\mathcal{L}^{2}(\mathbb{R}, d \mu)$

$$
A=\frac{1}{\sqrt{2}}\left(\sqrt{1+\lambda x^{2}} \frac{d}{d x}+\frac{\beta x}{\sqrt{1+\lambda x^{2}}}\right),
$$

for which its adjoint operator is

$$
A^{+}=\frac{1}{\sqrt{2}}\left(-\sqrt{1+\lambda x^{2}} \frac{d}{d x}+\frac{\beta x}{\sqrt{1+\lambda x^{2}}}\right) .
$$

Then, we find that if $\widehat{H}_{1}^{\prime}=\widehat{H}_{1}-(1 / 2) \beta$,

$$
\begin{aligned}
& \widehat{H}_{1}^{\prime}=A^{+} A=-\frac{1}{2}\left(1+\lambda x^{2}\right) \frac{d^{2}}{d x^{2}}-\frac{1}{2} \lambda x \frac{d}{d x}+\frac{1}{2} \beta(\beta+\lambda) \frac{x^{2}}{1+\lambda x^{2}}-\frac{1}{2} \beta, \\
& \widehat{H}_{2}^{\prime}=A A^{+}=-\frac{1}{2}\left(1+\lambda x^{2}\right) \frac{d^{2}}{d x^{2}}-\frac{1}{2} \lambda x \frac{d}{d x}+\frac{1}{2} \beta(\beta-\lambda) \frac{x^{2}}{1+\lambda x^{2}}+\frac{1}{2} \beta .
\end{aligned}
$$

This shows that there is one positive number $\beta$ for which the Hamiltonian $\widehat{H}_{1}^{\prime}$ of the quantum non-linear oscillator admits a factorization with the parameters $\alpha$ and $\beta$ related by $\alpha^{2}=\beta(\beta+\lambda)$.

In our preceding case, the parameter being $\beta$, when comparing $\widehat{H}_{1}^{\prime}$ with its partner, as

$$
\widehat{H}_{1}^{\prime}(\beta-\lambda)=-\frac{1}{2}\left[\left(1+\lambda x^{2}\right) \frac{d^{2}}{d x^{2}}+\lambda x \frac{d}{d x}\right]+\frac{1}{2}(\beta-\lambda) \beta\left(\frac{x^{2}}{1+\lambda x^{2}}\right)-\frac{1}{2}(\beta-\lambda),
$$

then we see that

$$
\widehat{H}_{1}^{\prime}(\beta-\lambda)=\left(\widehat{H}_{2}^{\prime}(\beta)-\frac{1}{2} \beta\right)-\frac{1}{2}(\beta-\lambda)
$$

and therefore

$$
\widehat{H}_{2}^{\prime}(\beta)=\widehat{H}_{1}^{\prime}(f(\beta))+\beta-\frac{1}{2} \lambda
$$

where $f$ is the function $f(\beta)=\beta-\lambda$. If $R$ is defined by $R(\beta)=\beta+(1 / 2)$, then

$$
\widehat{H}_{2}^{\prime}(\beta)=\widehat{H}_{1}^{\prime}\left(\beta_{1}\right)+R\left(\beta_{1}\right) .
$$

This shows that, as the quantum non-linear oscillator is shape invariant, we can develop the method sketched before: First, the eigenvector $\left|\Psi_{0}\right\rangle$ is determined by the condition $A\left(\beta_{0}\right)\left|\Psi_{0}\right\rangle=0$. More specifically, we should solve the differential equation

$$
\frac{d}{d x} \Psi_{0}+\beta \frac{x}{1+\lambda x^{2}} \Psi_{0}=0
$$

and therefore the wave function of the fundamental state must be proportional to

$$
\Psi_{0}=\frac{1}{\left(1+\lambda x^{2}\right)^{r_{0}}}, \quad r_{0}=\frac{\beta}{2 \lambda}
$$

The energies of the first excited states will be

$$
E_{1}^{\prime}=R\left(\beta_{1}\right)=\beta-\lambda+\frac{\lambda}{2}
$$


and iterating the process we get

$$
E_{n}^{\prime}=\sum_{k=1}^{n} R\left(\beta_{k}\right)=\sum_{k=1}^{n}\left(\beta_{k}+\frac{\lambda}{2}\right)=\sum_{k=1}^{n}\left(\beta-\lambda k+\frac{\lambda}{2}\right),
$$

and therefore,

$$
E_{n}^{\prime}=n \beta+\lambda\left[\frac{n}{2}-\sum_{k=1}^{n} k\right]=n \beta-\frac{n^{2}}{2} \lambda .
$$

The energy of the eigenstates of $\widehat{H}_{1}=\widehat{H}_{1}^{\prime}+(1 / 2) \beta$ will be given by

$$
E_{n}=n \beta-\frac{n^{2}}{2} \lambda+\frac{1}{2} \beta \text {. }
$$

The method also provides us the corresponding eigenfunctions as

$$
\begin{aligned}
& \left|\Psi_{1}(\beta)\right\rangle=A^{+}(\beta)\left|\Psi_{0}\left(\beta_{1}\right)\right\rangle, \\
& \ldots \ldots \ldots \ldots \ldots \ldots \ldots \ldots \ldots \ldots \\
& \left|\Psi_{n}(\beta)\right\rangle=A^{+}(\beta) A^{+}\left(\beta_{1}\right) \cdots A^{+}\left(\beta_{n-1}\right)\left|\Psi_{0}\left(\beta_{n}\right)\right\rangle .
\end{aligned}
$$

There is a clear difference between the case $\lambda>0$ and the case $\lambda<0$. Note that the lowest value for $E_{n}^{\prime}$ is $E_{0}^{\prime}=0$. Therefore: If $\lambda>0$ only values such that

$$
\beta-\lambda \frac{n}{2} \geq 0 \Longrightarrow n \leq \frac{2 \beta}{\lambda}
$$

are allowed. The eigenvalues are not equally spaced. On the contrary, when $\lambda<0$ all natural numbers are allowed for $n$

\section{Quantization of the 2-dimensional nonlinear harmonic oscillator}

There exist relatively few examples of quantum Hamiltonians in two dimensions whose spectrum can be fully determined by algebraic methods. We shall show that the deformed nonlinear oscillator is one of such examples. Furthermore, this can be done in different ways [44].

First, one can check that all the measures $d \mu=\rho(x, y) d x \wedge d y$ invariant under the vector fields

$$
X_{1}=\sqrt{1+\lambda r^{2}} \frac{\partial}{\partial x}, \quad X_{2}=\sqrt{1+\lambda r^{2}} \frac{\partial}{\partial y},
$$

are proportional to

$$
d \mu=\frac{1}{\sqrt{1+\lambda r^{2}}} d x \wedge d y .
$$

Therefore we shall consider the Hilbert space $\mathcal{L}^{2}\left(\mathbb{R}^{2}, d \mu\right)$.

The form of Killing vectors $X_{1}$ and $X_{2}$ generating 'translations' suggests us to take the momenta operators

$$
\widehat{P}_{x}=-i \hbar \sqrt{1+\lambda r^{2}} \frac{\partial}{\partial x}, \quad \widehat{P}_{y}=-i \hbar \sqrt{1+\lambda r^{2}} \frac{\partial}{\partial y}
$$


and then the quantum Hamiltonian is

$$
\begin{aligned}
\widehat{H}= & -\frac{\hbar^{2}}{2 m}\left(\left(1+\lambda r^{2}\right) \frac{\partial^{2}}{\partial x^{2}}+\lambda x \frac{\partial}{\partial x}\right)-\frac{\hbar^{2}}{2 m}\left(\left(1+\lambda r^{2}\right) \frac{\partial^{2}}{\partial y^{2}}+\lambda y \frac{\partial}{\partial y}\right) \\
& +\lambda \frac{\hbar^{2}}{2 m}\left(x^{2} \frac{\partial^{2}}{\partial y^{2}}+y^{2} \frac{\partial^{2}}{\partial x^{2}}-2 x y \frac{\partial^{2}}{\partial x \partial y}-x \frac{\partial}{\partial x}-y \frac{\partial}{\partial y}\right)+\frac{1}{2} g \frac{r^{2}}{1+\lambda r^{2}}
\end{aligned}
$$

which can be written as

$$
\widehat{H}=\widehat{H}_{1}+\widehat{H}_{2}-\lambda \widehat{J}^{2},
$$

with

$$
\begin{aligned}
& \widehat{H}_{1}=-\frac{\hbar^{2}}{2 m}\left(\left(1+\lambda r^{2}\right) \frac{\partial^{2}}{\partial x^{2}}+\lambda x \frac{\partial}{\partial x}\right)+\frac{1}{2} g \frac{x^{2}}{1+\lambda r^{2}} \\
& \widehat{H}_{2}=-\frac{\hbar^{2}}{2 m}\left(\left(1+\lambda r^{2}\right) \frac{\partial^{2}}{\partial y^{2}}+\lambda y \frac{\partial}{\partial y}\right)+\frac{1}{2} g \frac{y^{2}}{1+\lambda r^{2}}, \\
& \widehat{J}^{2}=-\frac{\hbar^{2}}{2 m}\left(x^{2} \frac{\partial^{2}}{\partial y^{2}}+y^{2} \frac{\partial^{2}}{\partial x^{2}}-2 x y \frac{\partial^{2}}{\partial x \partial y}-x \frac{\partial}{\partial x}-y \frac{\partial}{\partial y}\right)+\frac{1}{2} g \frac{r^{2}}{1+\lambda r^{2}}
\end{aligned}
$$

Note that each term commutes with the sum of the other two and therefore with $\widehat{H}$. Consequently, we can consider three different (complete) systems of compatible observables:

$$
\left\{\widehat{H}_{1}, \widehat{H}_{2}-\lambda J^{2}\right\}, \quad\left\{\widehat{H}_{1}-\lambda J^{2}, \widehat{H}_{2}\right\}, \quad\left\{\widehat{H}_{1}+\widehat{H}_{2}, J\right\} .
$$

Therefore we should solve one of these spectral problems:
A) $\widehat{H}_{1} \Psi\left(E_{1}, E_{2 j}\right)=E_{1} \Psi\left(E_{1}, E_{2 j}\right), \quad\left(\widehat{H}_{2}-\lambda J^{2}\right) \Psi\left(E_{1}, E_{2 j}\right)=E_{2 j} \Psi\left(E_{1}, E_{2 j}\right)$;
B) $\left(\widehat{H}_{1}-\lambda J^{2}\right) \Psi\left(E_{1 j}, E_{2}\right)=E_{1 j} \Psi\left(E_{1 j}, E_{2}\right), \quad \widehat{H}_{2} \Psi\left(E_{1 j}, E_{2}\right)=E_{2} \Psi\left(E_{1 j}, E_{2}\right)$;
C) $\widehat{H}_{1} \Psi\left(E_{1}, E_{2 j}\right)=E_{1} \Psi\left(E_{1}, E_{2 j}\right), \quad\left(\widehat{H}_{2}-\lambda J^{2}\right) \Psi\left(E_{1}, E_{2 j}\right)=E_{2 j} \Psi\left(E_{1}, E_{2 j}\right)$.

As in the one-dimensional case, it is convenient to use $g=m \alpha^{2}+\lambda \hbar \alpha$ and dimensionless variables

$$
x=\sqrt{\frac{\hbar}{m \alpha}} \tilde{x}, \quad y=\sqrt{\frac{\hbar}{m \alpha}} \tilde{y}, \quad \lambda=\frac{m \alpha}{\hbar} \Lambda, \quad E=\hbar \alpha e
$$

for which $1+\lambda r^{2}=1+\Lambda \tilde{r}^{2}$ and the Schrödinger equation becomes

$$
\begin{aligned}
& -\left(\left(1+\Lambda r^{2}\right) \frac{\partial^{2}}{\partial x^{2}}+\Lambda x \frac{\partial}{\partial x}\right) \Psi-\frac{\hbar^{2}}{2 m}\left(\left(1+\Lambda r^{2}\right) \frac{\partial^{2}}{\partial y^{2}}+\Lambda y \frac{\partial}{\partial y}\right) \Psi \\
& +\Lambda \frac{\hbar^{2}}{2 m}\left(x^{2} \frac{\partial^{2}}{\partial y^{2}}+y^{2} \frac{\partial^{2}}{\partial x^{2}}-2 x y \frac{\partial^{2}}{\partial x \partial y}-x \frac{\partial}{\partial x}-y \frac{\partial}{\partial y}\right) \Psi+\frac{1}{2}(1+\Lambda) \frac{r^{2}}{1+\Lambda r^{2}} \Psi=e \Psi .
\end{aligned}
$$

A) As the Hamilton-Jacobi equation separates in coordinates $\left(z_{x}, y\right)$ we shall use such coordinates to write the Schrödinger equation ( $z$ is used instead of $z_{x}$ )

$$
\begin{aligned}
-\frac{1}{2}( & \left.\frac{1+\Lambda z^{2}}{1+\Lambda y^{2}} \frac{\partial^{2}}{\partial z^{2}}+\frac{\Lambda z}{1+\Lambda y^{2}} \frac{\partial}{\partial z}\right) \Psi \\
& -\frac{1}{2}\left(\left(1+\Lambda y^{2}\right) \frac{\partial^{2}}{\partial y^{2}}+2 \Lambda y \frac{\partial}{\partial z}\right) \Psi+\frac{1}{2} \frac{1+\Lambda}{1+\Lambda y^{2}}\left(\frac{z^{2}}{1+\Lambda z^{2}}+y^{2}\right) \Psi=e \Psi,
\end{aligned}
$$


and assuming a factorization for $\Psi(z, y)$ of the form $\Psi(z, y)=Z(z) Y(y)$ we find the following equations:

$$
\begin{aligned}
-\frac{1}{2}( & \left.\left(1+\Lambda z^{2}\right) Z^{\prime \prime}+\Lambda z Z^{\prime}\right)+\frac{1}{2}(1+\Lambda) \frac{z^{2}}{1+\Lambda z^{2}} Z=\mu Z, \\
- & \frac{1}{2}\left(\left(1+\Lambda y^{2}\right)^{2} Y^{\prime \prime}+2 \Lambda y\left(1+\Lambda y^{2}\right) Y^{\prime}\right)+\left(\frac{1}{2}(1+\Lambda) y^{2}-\left(1+\Lambda y^{2}\right) e\right) Y=-\mu Y .
\end{aligned}
$$

In this way the two-dimensional problem has been decoupled in two 1-dimensional equations. The first one is the corresponding one to a 1-dimensional system. The second one however is a bit different because includes the contribution of the angular momentum.

If a new parameter $\nu=e-\mu$ is introduced the second equation becomes

$$
\left(1+\Lambda y^{2}\right) Y^{\prime \prime}+2 \Lambda y Y^{\prime}-(1+\Lambda-2 \Lambda \mu) \frac{y^{2}}{1+\Lambda y^{2}} Y+2 \nu Y=0
$$

i.e. defining $G_{\mu}^{2}=1+(1-2 \mu) \Lambda$,

$$
\left(1+\Lambda y^{2}\right) Y^{\prime \prime}+2 \Lambda y Y^{\prime}-G_{\mu}^{2} \frac{y^{2}}{1+\Lambda y^{2}} Y+2 \nu Y=0,
$$

and then writing

$$
Y(\Lambda, \mu)=q(y, \Lambda)\left(1+\Lambda y^{2}\right)^{-G_{\mu} /(2 \Lambda)},
$$

it becomes

$$
\left(1+\Lambda y^{2}\right) q^{\prime \prime}+2\left(\lambda-G_{\mu}\right) y q^{\prime}+\left(2 \nu-G_{\mu}\right) q=0
$$

which is a deformation of the Hermite equation.

Assuming the power expansion

$$
q(y, \Lambda)=\sum_{n=0}^{\infty} c_{n}(\Lambda) y^{n}
$$

we obtain the recursion relation

$$
c_{n+2}=-\frac{c_{n}}{(n+2)(n+1)}\left(\Lambda n(n-1)-G_{\mu}(2 n+1)+2 \nu\right) .
$$

Therefore the general solution is determined by the values of the coefficients $c_{0}$ and $c_{1}$. In particular we can write the solutions $q_{0}$ and $q_{1}$ determined by $c_{0}=1, c_{1}=0$ and $c_{0}=1, c_{1}=0$, respectively. The solution $q_{0}$ is an even function while the $q_{1}$ function is odd. The general solution is $q=c_{0} q_{0}+c_{1} q_{1}$.

The radius of convergence of both power series defining $q_{0}$ and $q_{1}$ is

$$
R=\frac{1}{\sqrt{|\Lambda|}}
$$

The solution reduces to a polynomial of degree $n$ if one of the coefficients is zero and there exists an integer number $n$ related with $\nu$ as follows:

$$
2 \nu=G_{\mu}(2 n+1)-n(n+1) \Lambda .
$$

One can study the properties of these polynomial solutions which have a lot of similarities with the Hermite polynomials. The associated Sturm-Liouville problem plays a relevant rôle. 
In summary, the bound states for this system have energies

$$
e_{m, n}=\mu_{m}+\nu_{n}=(m+n+1)\left(1-\frac{1}{2}(m+n) \lambda\right)
$$

and wave functions given by

$$
\Psi_{m, n}(z, y)=Z_{m}(z) Y_{n}(y), \quad z=\frac{x}{\sqrt{1+\Lambda y^{2}}}
$$

We finally mention that the properties of these $\lambda$-dependent Hermite polynomials are discussed in $[40,44]$. It is proved the orthogonality as well as the existence of a $\lambda$-dependent Rodrigues formula, a generating function and $\lambda$-dependent recursion relations between polynomials of different orders.

\section{Acknowledgments}

Partial financial support of research projects BFM-2003-02532, FPA-2003-02948, MTM-200509183, DGA E24/1 and VA013C05 is acknowledged.

\section{References}

[1] Bertrand M.J., Théorème relatif au mouvement d'un point attiré vers un centre fixe, C. R. Math. Acad. Sci. Paris LXXVII (1873), no. 16, 849-854.

[2] Fris T.I., Mandrosov V., Smorodinsky Y.A., Uhlir M., Winternitz P., On higher symmetries in quantum mechanics, Phys. Lett. 16 (1965), 354-356.

[3] Evans N.W., Superintegrability of the Smorodinsky-Winternitz system, Phys. Lett. 147 (1990), $483-486$.

[4] Evans N.W., Group theory of the Smorodinsky-Winternitz system, J. Math. Phys. 32 (1991), 3369-3375.

[5] Tempesta P.,Winternitz P. et al. (Editors), Super-integrability in classical and quantum systems, Proceedings of the Workshop held at the Université de Montréal, CRM Proc. Lecture Notes, Vol. 37, AMS, Providence, 2004.

[6] Kalnins E.G., Kress J.M., Miller W., Second-order superintegrable systems in conformally flat spaces. I. Two-dimensional classical structure theory, J. Math. Phys. 46 (2005), 053509, 28 pages.

[7] Kalnins E.G., Kress J.M., Miller W., Second order superintegrable systems in conformally flat spaces. II. The classical two-dimensional Stäckel transform, J. Math. Phys. 46 (2005), 053510, 15 pages.

[8] Błaszak M., Sergyeyev A., Maximal superintegrability of Benenti systems, J. Phys. A: Math. Gen. 38 (2005), L1-L5, nlin.SI/0412018.

[9] Daskaloyannis C., Ypsilantis K., Unified treatment and classification of superintegrable systems with integrals quadratic in momenta on a two-dimensional manifold, J. Math. Phys. 47 (2006), 042904, 38 pages, math-ph/0412055.

[10] Grosche C., Pogosyan G.S., Sissakian A.N., Path integral discussion for Smorodinsky-Winternitz potentials. II. Two- and three-dimensional sphere, Fortschr. Phys. 43 (1995), 523-563.

[11] Rañada M.F., Superintegrabe $n=2$ systems, quadratic constants of motion, and potentials of Drach, J. Math. Phys. 38 (1997), 4165-4178.

[12] Kalnins E.G., Miller W., Pogosyan G.S., Superintegrability on the two-dimensional hyperboloid, J. Math. Phys. 38 (1997), 5416-5433.

[13] Rañada M.F., Santander M., Superintegrable systems on the two-dimensional sphere $S^{2}$ and the hyperbolic plane $H^{2}$, J. Math. Phys. 40 (1999), 5026-5057.

[14] Kalnins E.G., Miller W., Pogosyan G.S., Coulomb-oscillator duality in spaces of constant curvature, J. Math. Phys. 41 (2000), 2629-2657, quant-ph/9906055.

[15] Slawianowski J.J., Bertrand systems on spaces of constant sectional curvature, Rep. Math. Phys. 46 (2000), 429-460.

[16] Kalnins E.G., Kress J.M., Pogosyan G.S., Miller W., Completeness of superintegrability in two-dimensional constant-curvature spaces, J. Phys. A: Math. Gen. 34 (2001), 4705-4720, math-ph/0102006. 
[17] Rañada M.F., Santander M., On the harmonic oscillator on the two-dimensional sphere $S^{2}$ and the hyperbolic plane $H^{2}$, J. Math. Phys. 43 (2002), 431-451.

[18] Kalnins E.G., Kress J.M., Winternitz P., Superintegrability in a two-dimensional space of nonconstant curvature, J. Math. Phys. 43 (2002), 970-983, math-ph/0108015.

[19] Ballesteros A., Herranz F.J., Santander M., Sanz-Gil T., Maximal superintegrability on $N$-dimensional curved spaces, J. Phys. A: Math. Gen. 36 (2003), L93-L99, math-ph/0211012.

[20] Rañada M.F., Santander M., On the harmonic oscillator on the two-dimensional sphere $S^{2}$ and the hyperbolic plane $H^{2}$ II, J. Math. Phys. 44 (2003), 2149-2167.

[21] Ballesteros A., Herranz F.J., Ragnisco O., Integrable potentials on spaces with curvature from quantum groups, J. Phys. A: Math. Gen. 38 (2005), 7129-7144, math-ph/0505081.

[22] Herranz F.J., Ballesteros A., Superintegrability on three-dimensional Riemannian and relativistic spaces of constant curvature, SIGMA 2 (2006), 010, 22 pages, math-ph/0512084.

[23] Mathews P.M., Lakshmanan M., On a unique nonlinear oscillator, Quart. Appl. Math. 32 (1974), $215-218$.

[24] Lakshmanan M., Rajasekar S., Nonlinear dynamics. Integrability, chaos and patterns, Adv. Texts Phys., Springer-Verlag, Berlin, 2003.

[25] Cariñena J.F., Rañada M.F., Santander M., Senthilvelan M., A non-linear oscillator with quasi-harmonic behaviour: two- and n-dimensional oscillators, Nonlinearity 17 (2004), 1941-1963, math-ph/0406002.

[26] Cariñena J.F., Rañada M.F., Santander M., A nonlinear deformation of the isotonic oscillator and the Smorodinski-Winternitz system: integrability and superintegrability, Regul. Chaotic Dyn. 10 (2005), 423436.

[27] Cariñena J.F., Rañada M.F., Santander M., Three superintegrable two-dimensional oscillators: super-integrability, nonlinearity and curvature, Physics of Atomic Nuclei 70 (2007), 505-512.

[28] Calogero F., Solution of a three body problem in one dimension, J. Math. Phys. 10 (1969), 2191-2196.

[29] Perelomov A.M., Integrable systems of classical mechanics and Lie algebras, Birkhäuser, 1990.

[30] Chalykh O.A., Veselov A.P., A remark on rational isochronous potentials, J. Nonlinear Math. Phys. 12 (2005), suppl. 1, 179-183, math-ph/0409062.

[31] Asorey M., Cariñena J.F., Marmo G., Perelomov A.M., Isoperiodic classical systems and their quantum counterparts, Ann. Phys. 322 (2007), to appear.

[32] Pinney E., The nonlinear differential equation $y^{\prime \prime}+p(x) y+c y^{-3}=0$, Proc. Amer. Math. Soc. 1 (1950), 681-681.

[33] Ermakov V., Second order differential equations. Conditions of complete integrability, Univ. Isz. Kiev Series III 9 (1880), 1-25 (translation by A.O. Harin).

[34] Dongpei Z., A new potential with the spectrum of an isotonic oscillator, J. Phys. A: Math. Gen. 20 (1987), 4331-4336.

[35] Cariñena J.F., Marmo G, Rañada M.F., Non-symplectic symmetries and bi-Hamiltonian structures of the rational harmonic oscillator, J. Phys. A: Math. Gen. 35 (2002), L679-L686, hep-th/0210260.

[36] Higgs P.W., Dynamical symmetries in a spherical geometry I, J. Phys. A: Math. Gen. 12 (1979), 309-323.

[37] Bonatsos D., Daskaloyannis C., Kokkotas K., Quantum-algebraic description of quantum superintegrable systems in two dimensions, Phys. Rev. A 48 (1993), R3407-R3410.

[38] Bonatsos D., Daskaloyannis C., Kokkotas K., Deformed oscillator algebras for two-dimensional quantum superintegrable systems, Phys. Rev. A 50 (1994), 3700-3709, hep-th/9309088.

[39] Cariñena J.F., Rañada M.F., Santander M., One-dimensional model of a quantum nonlinear harmonic oscillator, Rep. Math. Phys. 54 (2004), 285-293, hep-th/0501106.

[40] Cariñena J.F., Rañada M.F., Santander M., A quantum exactly solvable nonlinear oscillator with quasiharmonic behaviour, Ann. Phys. 322 (2007), 434-459, math-ph/0604008.

[41] Cariñena J.F., Ramos A., Riccati equation, factorization method and shape invariance, Rev. Math. Phys. 12 (2000), 1279-1304, math-ph/9910020.

[42] Gendenshteïn L.É., Derivation of exact spectra of the Schrödinger equation by means of supersymmetry, JETP Lett. 38 (1983), 356-359.

[43] Gendenshteïn L.É., Krive I.V., Supersymmetry in quantum mechanics, Soviet Phys. Uspekhi 28 (1985), 645-666.

[44] Cariñena J.F., Rañada M.F., Santander M., The quantum harmonic oscillator on the sphere and the hyperbolic plane, Ann. Phys. 322 (2007), to appear. 